\title{
Motivos para o Perfeccionismo e Intolerância à Frustração nos Estudantes de Medicina da Universidade de Coimbra
}

\section{Reasons for Perfectionism and Intolerance to Frustration in Medicine Students of University of Coimbra}

\author{
Sara BATISTA $\bowtie 1$, Luiz Miguel SANTIAGO1', Inês ROSENDO1 \\ Acta Med Port 2018 Oct;31(10):527-533 - https://doi.org/10.20344/amp.9976
}

\begin{abstract}
RESUMO
Introdução: O perfeccionismo e a intolerância à frustração são os principais fatores de vulnerabilidade ao stress psicológico nos estudantes do Mestrado Integrado em Medicina da Faculdade de Medicina da Universidade de Coimbra. Este estudo procurou investigar a causalidade dos mesmos para sua prevenção.

Material e Métodos: Estudo observacional em triangulação, qualitativo-quantitativo, com inventariação dos principais motivos em painel de alunos, sendo a listagem obtida organizada e colocada em questionário complementado com inquérito epidemiológico, aplicado online a todos os alunos do Mestrado Integrado em Medicina da Faculdade de Medicina da Universidade de Coimbra, seguindo-se análise estatística.
\end{abstract}

Resultados: Estudou-se amostra representativa, de $n=368,77,7 \%$ do sexo feminino. Os principais motivos para perfeccionismo e intolerância à frustração são 'fatores intrínsecos' e 'exigências da profissão médica', com, respetivamente, 91,1\% e 91,8\% de respostas 'importante'/'muito importante'; 'pressão ambiental' é o menos importante, com $68,2 \%$ em tais classificações. Alunos satisfeitos com a vida curricular atribuem menor importância à 'pressão ambiental' $(p=0,004)$, 'insegurança quanto à formação profissional' $(p=0,017)$, 'métodos de avaliação curricular' $(p=0,002)$ e 'exigências curriculares do Mestrado Integrado em Medicina' $(p=0,002)$; o sexo feminino dá maior importância às 'exigências curriculares do Mestrado Integrado em Medicina' $(p=0,001)$; alunos inseridos em atividade extracurricular, consideram menos importante a 'pressão ambiental' $(p=0,007)$.

Discussão: Nesta amostra a vulnerabilidade ao stress psicológico por perfeccionismo e intolerância à frustração depende essencialmente da personalidade auto-exigente. Outro importante fator corresponde à insegurança relativamente às exigências profissionais, com sofrimento por antecipação e ausência de perspetiva profissional.

Conclusão: O suporte psicológico, o envolvimento em atividades extracurriculares específicas e a reestruturação curricular parecem ser formas de diminuir a vulnerabilidade ao stress nos estudantes de medicina.

Palavras-chave: Educação Médica; Estudantes, Medicina; Frustração; Perfeccionismo; Stress Psicológico

\section{ABSTRACT}

Introduction: Perfectionism and intolerance to frustration are the main factors of vulnerability to psychological stress observed in students of the Integrated Master Degree in Medicine of the Faculty of Medicine, University of Coimbra, a study claims. We aimed to ascertain their reasons, seeking for their prevention.

Material and Methods: An observational triangulation study was performed, collecting the main reasons according to the opinion of a students focus-group, organized in a questionnaire completed with epidemiologic data, applied online to all students of the Integrated Master Degree in Medicine of the Faculty of Medicine, University of Coimbra. Statistical analysis was performed.

Results: A representative sample, of $n=368,77.7 \%$ female, was studied. The most important responded reasons were 'intrinsic factors' and 'medical profession demands', with, respectively, $91.1 \%$ and $91.8 \%$ of 'important'/'very important' answers; 'environmental pressure' is the less important, with $68.2 \%$ attributing those classifications. Students satisfied with curricular life attribute less importance to 'environmental pressure' $(p=0.004)$, 'insecurity about professional training' $(p=0.017)$, 'curricular evaluation methods' $(p=0.002)$ and 'Integrated Master Degree in Medicine of the Faculty of Medicine, University of Coimbra curricular demands' $(p=0.002)$; female students assign more importance to 'Integrated Master Degree in Medicine curricular demands' $(p=0.001)$; students involved in an extracurricular activity consider less important the 'environmental pressure' $(p=0.007)$.

Discussion: In this Integrated Master Degree in Medicine of the Faculty of Medicine, University of Coimbra students sample, vulnerability to psychological stress associated to perfectionism and intolerance to frustration is due essentially to self-demanding personality. Insecurity about professional demands, associated to suffering in anticipation and the absence of professional perspectives, represent another important cause.

Conclusion: Psychological support, involvement in specific extracurricular activities and curricular reorganisation appear to be means of reducing the vulnerability to stress in medical students.

Keywords: Education, Medical; Frustration; Perfectionism; Stress, Psychological; Students, Medical

\section{INTRODUÇÃO}

Níveis importantes de ansiedade estão patentes desde as fases mais precoces da educação médica, fazendo crer que os estudantes se encontram desde cedo sob o peso de fatores stressantes que poderão, mais tarde, ser fonte de exaustão e burnout, levando a comprometimento da capacidade cognitiva dos alunos. ${ }^{1-12}$ Pode ainda surgir mor- bilidade psicossocial, em que se inserem distúrbios de ansiedade, abuso de álcool e outras substâncias, depressão e ideação suicida. ${ }^{1,4,5,8,9,11,13}$ São ainda evidentes as consequências na prática clínica, com prejuízo da prestação de cuidados e de profissionalismo médico. ${ }^{4,9,11}$

Torna-se assim premente o estudo das diversas fontes

1. Clínica Universitária de Medicina Geral e Familiar. Faculdade de Medicina. Universidade de Coimbra. Coimbra. Portugal.

$\triangle$ Autor correspondente: Sara Batista. sarageadabatista@gmail.com

Recebido: 25 de novembro de 2017 - Aceite: 16 de agosto de 2018 | Copyright @ Ordem dos Médicos 2018 
de stress a que esta população está sujeita, considerando-se essencial que as escolas médicas tomem, como prioridade, a promoção do bem-estar dos estudantes que frequentam a sua academia, ${ }^{1,2,5,13}$ com aplicação de estratégias preventivas logo numa fase precoce do ensino. ${ }^{4,8}$

Neste sentido, foi desenvolvido um estudo por Pereira APM ${ }^{14}$ destinado a avaliar a vulnerabilidade ao stress nos estudantes dos quinto e sexto anos do Mestrado Integrado em Medicina da Faculdade de Medicina da Universidade de Coimbra (MIM-FMUC), no ano letivo 2015/2016, com recurso à escala 23-QVS. ${ }^{15}$ Como conclusões do estudo destaca-se que "dos alunos avaliados, 25,2\% encontram-se vulneráveis ao stress, sendo a dimensão do perfeccionismo e intolerância à frustração a mais afetada", ${ }^{14}$ o que vai ao encontro da personalidade tipicamente perfeccionista presente em quem ingressa na carreira médica. ${ }^{16}$ Porque a vulnerabilidade ao stress se verifica desde os primeiros anos do curso, sendo crítico o período de adaptação entre a transição do ensino secundário para o ensino superior, ${ }^{2,4-6}$ o presente estudo dirigiu-se a todos os anos do MIM da FMUC. Isto permitirá não só intervir precocemente, como também objetivar diferenças existentes entre as várias fases do curso de medicina, onde os estudantes passam por diversas etapas e lidam com diferentes fontes de stress. ${ }^{4,8,11}$ Por outro lado, diversos estudos têm estudado variáveis sociodemográficas que possam apresentar relação com a vulnerabilidade ao stress em alunos, ${ }^{2,3,9,14,17}$ de modo a facilitar o desenvolvimento de estratégias de coping mais dirigidas. ${ }^{2,4,5,8}$

O presente trabalho tem como objetivo geral identificar as razões que justificam tão elevados níveis de perfeccionismo e intolerância à frustração nos estudantes do MIM-FMUC. Procuraram-se ainda reconhecer diferenças em função do sexo, da inserção em atividade extracurricular e da satisfação com a vida curricular, no que respeita à importância atribuída a cada motivo.

\section{MATERIAL E MÉTODOS}

Conduziu-se uma metodologia observacional em triangulação com estudo qualitativo e quantitativo em várias fases.

A primeira fase, qualitativa, decorreu em abril de 2017 e consistiu na solicitação da participação, informal e voluntária, de dois alunos do sexo feminino e um do sexo masculino de cada ano de frequência do MIM-FMUC, para nomeação de dois motivos que justificassem o perfeccionismo e intolerância à frustração verificados nessa população. A seleção destes alunos foi realizada pelas comissões de curso de cada ano do MIM-FMUC, a quem solicitámos nomes e contactos de alunos a convidar para a tarefa, que foram contactados assim que possível.

A segunda fase, de análise e organização das respostas obtidas, realizadas pelos autores, resultou num conjunto de frases que representam o resumo das ideias expressas. Tais frases foram organizadas numa lista de motivos, na qual se baseou a elaboração do questionário, com resposta em escala de tipo Likert, para classificação de cada motivo como 'nada importante', 'pouco importante', 'importante' ou 'muito importante'. Foi ainda incluído um campo para resposta aberta, destinado a indicar outro motivo que não constasse naqueles reunidos. Do questionário constava também um inquérito sobre os seguintes dados epidemiológicos: ano de frequência do MIM-FMUC, idade, sexo, satisfação com a vida curricular na maior parte do tempo e inserção em atividade extracurricular. Antes de aplicado, o questionário foi divulgado a seis estudantes do quinto ano do MIM-FMUC, de forma a obter validação quanto à sua clareza, compreensão, organização e estrutura gramatical, tendo-se obtido opinião positiva, sem sugestão de alterações.

A fase terceira, de estudo quantitativo com intenção analítica, decorreu pela aplicação do questionário à população, em preenchimento voluntário, anónimo e confidencial, solicitando-se consentimento informado requerido para participação no estudo. Foi garantida a inexistência de respostas duplicadas, com o pedido dos últimos dois dígitos do cartão de cidadão, a última letra do apelido e o dia de nascimento (não indicando o mês nem o ano).

O questionário foi construído e aplicado em Google Drive, tendo-se responsabilizado pela sua divulgação as comissões de curso dos seis anos de frequência do MIM-FMUC. Esta divulgação decorreu entre 1 e 30 de junho de 2017, no grupo que cada ano de curso possui na rede social Facebook. A base de dados foi encerrada a 30 de junho de 2017.

O cálculo do tamanho da amostra foi efetuado em função do universo dos alunos que frequentavam o MIM-FMUC no ano letivo de 2016/2017 ( $n=1945)$, obtendo-se uma amostra necessária de 277 alunos.

Os dados obtidos no questionário foram registados em base de Microsoft Excel e, com recurso ao programa Statistical Package for the Social Sciences (SPSS - PASW $18)$, foram alvo de análise estatística descritiva e inferencial, tendo-se utilizado, na primeira, a média e desvio padrão para caraterização das variáveis quantitativas, sendo as variáveis qualitativas descritas segundo frequências absolutas e relativas. A análise inferencial, para variáveis ordinais foi realizada com recurso ao teste $t$ de Student, quando as variáveis tinham distribuição normal (teste de Kolmogorov-Smirnov), e pelo teste $U$ de Mann-Whitney. Para variáveis nominais utilizou-se o teste qui-quadrado $(\chi 2)$, sendo sempre definido o valor de $p<0,05$ como estatisticamente significativo.

Este estudo obteve o parecer favorável da Comissão de Ética da Administração Regional de Saúde do Centro, Instituto Público.

\section{RESULTADOS}

\section{Estudo qualitativo}

Após análise das 36 respostas obtidas na primeira fase, de estudo qualitativo, foram formulados os motivos abaixo, que explicam cada um dos ambientes arbitrariamente nomeados pelos autores (Tabela 1). 
Tabela 1 - Motivos para o perfeccionismo e intolerância à frustração nos estudantes do MIM-FMUC

\begin{tabular}{ll}
\hline Motivos & Descrição \\
\hline Fatores intrínsecos & $\begin{array}{l}\text { Perfeccionismo individual; incapacidade de lidar adequadamente com o } \\
\text { falhanço; personalidade narcisista; autoexigência. }\end{array}$ \\
Exigências da profissão médica & $\begin{array}{l}\text { Perceção de não haver lugar para o erro; medo de não ser capaz de } \\
\text { exercer corretamente a profissão médica. }\end{array}$ \\
Pressão ambiental & Exercida pela sociedade, família, pares, escolas, professores. \\
Incerteza quanto ao futuro pretendido & $\begin{array}{l}\text { Redução do número de vagas de especialidade; perceção da } \\
\text { necessidade de ter que ser melhor que os pares. }\end{array}$ \\
Insegurança sobre a formação profissional & Pouca prática clínica existente ao longo do curso. \\
Métodos de avaliação curricular & Avaliações não uniformes com notas diferentes em ambientes diferentes. \\
Exigências curriculares do MIM & A falta de tempo para o próprio. \\
\hline
\end{tabular}

Tabela 2 - Variáveis epidemiológicas medidas por ano de frequência do MIM da FMUC no ano letivo de 2016/2017

\begin{tabular}{|c|c|c|c|c|c|c|c|}
\hline & $\begin{array}{c}1^{\circ} \text { ano } \\
\mathrm{n}(\%)\end{array}$ & $\begin{array}{c}2^{\circ} \text { ano } \\
\mathrm{n}(\%)\end{array}$ & $\begin{array}{c}3^{\circ} \text { ano } \\
\text { n (\%) }\end{array}$ & $\begin{array}{c}4^{\circ} \text { ano } \\
\text { n (\%) }\end{array}$ & $\begin{array}{c}5^{\circ} \text { ano } \\
\mathrm{n}(\%)\end{array}$ & $\begin{array}{l}6^{\circ} \text { ano } \\
\text { n (\%) }\end{array}$ & $\begin{array}{l}\text { Total } \\
\text { n (\%) }\end{array}$ \\
\hline \multicolumn{8}{|l|}{ Sexo * } \\
\hline Feminino & $39(79,6)$ & $54(87,1)$ & $53(76,8)$ & $49(76,6)$ & $47(68,1)$ & $44(80,0)$ & $286(77,7)$ \\
\hline Masculino & $10(20,4)$ & $8(12,9)$ & $16(23,2)$ & $15(23,4)$ & $22(31,9)$ & $11(20,0)$ & $82(22,3)$ \\
\hline \multicolumn{8}{|c|}{ Satisfação com a vida curricular ** } \\
\hline $\operatorname{Sim}$ & $35(71,4)$ & $37(59,7)$ & $40(58,0)$ & $44(68,8)$ & $47(68,1)$ & $42(76,4)$ & $245(66,6)$ \\
\hline Não & $14(28,6)$ & $25(40,3)$ & $29(42,0)$ & $20(31,3)$ & $22(31,9)$ & $13(23,6)$ & $123(33,4)$ \\
\hline \multicolumn{8}{|c|}{ Inserção em atividade extracurricular *** } \\
\hline $\operatorname{Sim}$ & $17(34,7)$ & $36(58,1)$ & $36(52,2)$ & $45(70,3)$ & $45(65,2)$ & $30(54,5)$ & $209(56,8)$ \\
\hline Não & $32(65,3)$ & $26(41,9)$ & $33(47,8)$ & $19(29,7)$ & $24(34,8)$ & $25(45,5)$ & $159(43,2)$ \\
\hline Total & $49(13,3)$ & $62(16,8)$ & $69(18,8)$ & $64(17,4)$ & $69(18,8)$ & $55(14,9)$ & $368(100)$ \\
\hline $\begin{array}{l}\text { Idade (em anos) } \\
\text { (média } \pm \text { desvio padrão) }\end{array}$ & $19,24 \pm 1,35$ & $19,71 \pm 1,08$ & $21,33 \pm 1,44$ & $22,66 \pm 1,84$ & $23,43 \pm 2,12$ & $24,25 \pm 1,66$ & $21,84 \pm 2,41$ \\
\hline
\end{tabular}

\section{Estudo quantitativo}

\section{Variáveis epidemiológicas}

No estudo quantitativo (Tabela 2), foi estudada uma amostra de $n=368$ alunos, com idade média de 21,8 $\pm 2,41$ anos (intervalo de confiança a $95 \%$ de 21,6 a 22,1 anos), idade mínima de 18 anos e máxima de 33 anos. Obteve-se uma amostra maioritariamente feminina, $n=286(77,7 \%)$, sendo de $33,4 \%$ a proporção dos não satisfeitos com a vida curricular e de 56,8\% a percentagem dos inseridos em alguma atividade extracurricular.

Atendendo à distribuição por anos de frequência do MIM-FMUC, obtiveram-se 49 respostas do primeiro ano, 62 do segundo ano, 69 do terceiro ano, 64 do quarto ano, 69 do quinto ano e 55 do sexto ano, o que dá uma proporção de respostas face aos alunos inscritos no ano letivo de $2016 / 2017$ de $12 \%, 22 \%, 23 \%$, 21\%, 20\% e 17\%, respetivamente. Apenas se obteve diferença significativa entre o ano de curso frequentado e a inserção numa atividade extracurricular, destacando-se a percentagem de alunos inseridos numa atividade extracurricular de $34,7 \%$ no primeiro ano do curso, $70,3 \%$ no quarto ano e $54,5 \%$ no sexto ano.

Motivos para o perfeccionismo e intolerância à frustração

Segundo a Tabela 3, os motivos aos quais foi atribuída maior importância pela amostra, com a soma das percentagens de 'importante' e 'muito importante' superior a $90 \%$, foram: 'fatores intrínsecos': $91,1 \%$, e 'exigências da profissão médica': 91,8\%. O motivo 'pressão ambiental' foi o que demonstrou representar menor importância, com 68,2\% de respostas 'importante' e/ou 'muito importante'.

Motivos para o perfeccionismo e intolerância à frustração em função das variáveis epidemiológicas.

$\mathrm{O}$ estudo dos motivos para o perfeccionismo e intolerância à frustração em função das variáveis epidemiológicas, permite verificar diferenças significativas segundo 'satisfação com a vida curricular', 'inserção em atividade extracurricular' e 'sexo'.

$\mathrm{Na}$ Tabela 4, observa-se que os alunos satisfeitos com a vida curricular atribuem menor importância à 'pressão ambiental', com $24,9 \%$ classificando esse motivo como 'muito importante', contra $38,2 \%$ dos alunos não satisfeitos a considerar esse fator 'muito importante'. Para 'insegurança quanto à formação profissional', 'métodos de avaliação curricular' e 'exigências curriculares do MIM', é também atribuído um menor grau de importância pelos alunos que afirmam estar satisfeitos com a sua vida curricular na maior parte do tempo (Tabela 4). Já no que respeita aos 'fatores intrínsecos', 'exigências da profissão médica' e 'incerteza 
quanto ao futuro pretendido', não se verificam diferenças significativas.

Quanto à análise das variáveis sexo e exigências curriculares do MIM, constata-se (Tabela 5) que uma maior percentagem de elementos do sexo feminino considera este motivo 'importante' $(39,2 \%$ contra $32,9 \%$ no sexo masculino) ou 'muito importante' (47,9\% contra $35,4 \%$ no sexo masculino), tendo uma maior proporção de elementos do sexo masculino atribuído a classificação de 'nada importante' $(6,1 \%$ contra $0,7 \%$ no sexo feminino) ou 'pouco impor- tante' ( $25,6 \%$ contra $12,2 \%$ no sexo feminino).

Ao analisar os motivos em função da inserção em atividade extracurricular, verificou-se existir relação estatisticamente significativa entre essa e a 'pressão ambiental", com um maior número de respostas 'importante' ou 'muito importante' entre aqueles que não praticam qualquer atividade extracurricular: $78,6 \%$ vs $61,7 \%$ dos que estão inseridos numa atividade extracurricular (Tabela 6).

Cinco participantes preencheram ainda o campo de resposta aberta do questionário, verificando-se, depois de

Tabela 3 - Os motivos enumerados e sua classificação por ano de frequência do MIM da FMUC no ano letivo de 2016/2017

\begin{tabular}{|c|c|c|c|c|c|c|c|}
\hline & $\begin{array}{c}1^{\circ} \text { ano } \\
\mathrm{n}(\%)\end{array}$ & $\begin{array}{c}2^{\circ} \text { ano } \\
\text { n (\%) }\end{array}$ & $\begin{array}{c}3^{\circ} \text { ano } \\
\text { n (\%) }\end{array}$ & $\begin{array}{c}4^{\circ} \text { ano } \\
\text { n (\%) }\end{array}$ & $\begin{array}{c}5^{\circ} \text { ano } \\
\text { n (\%) }\end{array}$ & $\begin{array}{l}6^{\circ} \text { ano } \\
\text { n (\%) }\end{array}$ & $\begin{array}{l}\text { Total } \\
\text { n (\%) }\end{array}$ \\
\hline \multicolumn{8}{|l|}{ Fatores intrínsecos } \\
\hline 1 - Nada importante & $1(2,0)$ & $1(1,6)$ & $0(0,0)$ & $2(3,1)$ & $0(0,0)$ & $1(1,8)$ & $5(1,4)$ \\
\hline 2 - Pouco importante & $3(6,1)$ & $2(3,2)$ & $8(11,6)$ & $3(4,7)$ & $5(7,2)$ & $7(12,7)$ & $28(7,6)$ \\
\hline 3 - Importante & $20(40,8)$ & $25(40,3)$ & $30(43,5)$ & $24(37,5)$ & $25(36,2)$ & $19(34,5)$ & $143(38,9)$ \\
\hline 4 - Muito Importante & $25(51,0)$ & $34(54,8)$ & $31(44,9)$ & $35(54,7)$ & $39(56,5)$ & $28(50,9)$ & $192(52,2)$ \\
\hline \multicolumn{8}{|c|}{ Exigências da profissão médica } \\
\hline 1 - Nada importante & $0(0,0)$ & $0(0,0)$ & $2(2,9)$ & $1(1,6)$ & $0(0,0)$ & $0(0,0)$ & $3(0,8)$ \\
\hline 2 - Pouco importante & $3(6,1)$ & $3(4,8)$ & $5(7,2)$ & $0(0,0)$ & $10(14,5)$ & $6(10,9)$ & $27(7,3)$ \\
\hline 3 - Importante & $18(36,7)$ & $22(35,5)$ & $20(29,0)$ & $24(37,5)$ & $22(31,9)$ & $24(43,6)$ & $130(35,3)$ \\
\hline 4 - Muito importante & $28(57,1)$ & $37(59,7)$ & $42(60,9)$ & $39(60,9)$ & $37(53,6)$ & $25(45,5)$ & $208(56,5)$ \\
\hline \multicolumn{8}{|l|}{ Pressão ambiental } \\
\hline 1 - Nada importante & $1(2,0)$ & $3(4,8)$ & $2(2,9)$ & $5(7,8)$ & $6(8,7)$ & $3(5,5)$ & $20(5,4)$ \\
\hline 2 - Pouco importante & $17(34,7)$ & $13(21,0)$ & $23(33,3)$ & $13(20,3)$ & $17(24,6)$ & $14(25,5)$ & $97(26,4)$ \\
\hline 3 - Importante & $16(32,7)$ & $27(43,5)$ & $27(39,1)$ & $22(34,4)$ & $29(42,0)$ & $22(40,0)$ & $143(38,9)$ \\
\hline 4 - Muito importante & $15(30,6)$ & $19(30,6)$ & $17(24,6)$ & $24(37,5)$ & $17(24,6)$ & $16(29,1)$ & $108(29,3)$ \\
\hline \multicolumn{8}{|c|}{ Incerteza quanto ao futuro pretendido } \\
\hline 1 - Nada importante & $1(2,0)$ & $0(0,0)$ & $0(0,0)$ & $4(6,3)$ & $2(2,9)$ & $0(0,0)$ & $7(1,9)$ \\
\hline 2 - Pouco importante & $7(14,3)$ & $7(11,3)$ & $9(13,0)$ & $7(10,9)$ & $10(14,5)$ & $9(16,4)$ & $49(13,3)$ \\
\hline 3 - Importante & $19(38,8)$ & $21(33,9)$ & $30(43,5)$ & $19(29,7)$ & $14(20,3)$ & $19(34,5)$ & $122(33,2)$ \\
\hline 4 - Muito importante & $22(44,9)$ & $34(54,8)$ & $30(43,5)$ & $34(53,1)$ & $43(62,3)$ & $27(49,1)$ & $190(51,6)$ \\
\hline \multicolumn{8}{|c|}{ Insegurança quanto à formação profissional } \\
\hline 1 - Nada importante & $0(0,0)$ & $1(1,6)$ & $2(2,9)$ & $2(3,1)$ & $2(2,9)$ & $1(1,8)$ & $8(2,2)$ \\
\hline 2 - Pouco importante & $11(22,4)$ & $13(21,0)$ & $11(15,9)$ & $10(15,6)$ & $9(13,0)$ & $10(18,2)$ & $64(17,4)$ \\
\hline 3 - Importante & $21(42,9)$ & $23(37,1)$ & $26(37,7)$ & $20(31,3)$ & $16(23,2)$ & $20(36,4)$ & $126(34,2)$ \\
\hline 4 - Muito importante & $17(34,7)$ & $25(40,3)$ & $30(43,5)$ & $32(50,0)$ & $42(60,9)$ & $24(43,6)$ & $170(46,2)$ \\
\hline \multicolumn{8}{|c|}{ Métodos de avaliação curricular } \\
\hline 1 - Nada importante & $0(0,0)$ & $0(0,0)$ & $2(2,9)$ & $3(4,7)$ & $2(2,9)$ & $1(1,8)$ & $8(2,2)$ \\
\hline 2 - Pouco importante & $3(6,1)$ & $14(22,6)$ & $12(17,4)$ & $11(17,2)$ & $11(15,9)$ & $12(21,8)$ & $63(17,1)$ \\
\hline 3 - Importante & $22(44,9)$ & $22(35,5)$ & $25(36,2)$ & $21(32,8)$ & $24(34,8)$ & $23(41,8)$ & $137(37,2)$ \\
\hline 4 - Muito importante & $24(49,0)$ & $26(41,9)$ & $30(43,5)$ & $29(45,3)$ & $32(46,4)$ & $19(34,5)$ & $160(43,5)$ \\
\hline \multicolumn{8}{|c|}{ Exigências curriculares do MIM } \\
\hline 1 - Nada importante & $1(2,0)$ & $0(0,0)$ & $1(1,4)$ & $1(1,6)$ & $3(4,3)$ & $1(1,8)$ & $7(1,9)$ \\
\hline 2 - Pouco importante & $3(6,1)$ & $9(14,5)$ & $9(13,0)$ & $12(18,8)$ & $11(15,9)$ & $12(21,8)$ & $56(15,2)$ \\
\hline 3 - Importante & $15(30,6)$ & $29(46,8)$ & $35(50,7)$ & $21(32,8)$ & $23(33,3)$ & $16(29,1)$ & $139(37,8)$ \\
\hline 4 - Muito importante & $30(61,2)$ & $24(38,7)$ & $24(34,8)$ & $30(46,9)$ & $32(46,4)$ & $26(47,3)$ & $166(45,1)$ \\
\hline
\end{tabular}


Tabela 4 - Pressão ambiental, insegurança quanto à formação profissional, métodos de avaliação curricular e exigências curriculares do MIM em função da satisfação com a vida curricular

\begin{tabular}{|c|c|c|}
\hline & \multicolumn{2}{|c|}{ Satisfação com a vida curricular } \\
\hline & Sim & Não \\
\hline & n (\%) & n (\%) \\
\hline \multicolumn{3}{|l|}{ Pressão ambiental * } \\
\hline 1 - Nada importante & $16(6,5)$ & $4(3,3)$ \\
\hline 2 - Pouco importante & $71(29,0)$ & $26(21,1)$ \\
\hline 3 - Importante & $97(39,6)$ & $46(37,4)$ \\
\hline 4 - Muito importante & $61(24,9)$ & $47(38,2)$ \\
\hline \multicolumn{3}{|c|}{ Insegurança quanto à formação profissional ** } \\
\hline 1 - Nada importante & $6(2,4)$ & $2(1,6)$ \\
\hline 2 - Pouco importante & $45(18,4)$ & $19(15,4)$ \\
\hline 3 - Importante & $93(38,0)$ & $33(26,8)$ \\
\hline 4 - Muito importante & $101(41,2)$ & $69(56,1)$ \\
\hline \multicolumn{3}{|c|}{ Métodos de avaliação curricular *** } \\
\hline 1 - Nada importante & $7(2,9)$ & $1(0,8)$ \\
\hline 2 - Pouco importante & $49(20,0)$ & $14(11,4)$ \\
\hline 3 - Importante & $95(38,8)$ & $42(34,1)$ \\
\hline 4 - Muito importante & $94(38,4)$ & $66(53,7)$ \\
\hline \multicolumn{3}{|c|}{ Exigências curriculares do MIM $\S$} \\
\hline 1 - Nada importante & $5(2,0)$ & $2(1,6)$ \\
\hline 2 - Pouco importante & $43(17,6)$ & $13(10,6)$ \\
\hline 3 - Importante & $103(42,0)$ & $36(29,3)$ \\
\hline 4 - Muito importante & $94(38,4)$ & $72(58,5)$ \\
\hline
\end{tabular}

${ }^{*}: p=0,004 ;{ }^{* *}: p=0,017 ;{ }^{* *}: p=0,002 ; \S: p=0,002$

Tabela 5 - Exigências curriculares do MIM segundo o Sexo

\begin{tabular}{ccc}
\hline & \multicolumn{2}{c}{ Sexo } \\
& Feminino & Masculino \\
& $\mathbf{n}(\%)$ & $\mathbf{n}(\%)$ \\
\hline Exigências curriculares do MIM * & & \\
\hline 1 - Nada importante & $2(0,7)$ & $5(6,1)$ \\
2 - Pouco importante & $35(12,2)$ & $21(25,6)$ \\
3 - Importante & $112(39,2)$ & $27(32,9)$ \\
4 - Muito importante & $137(47,9)$ & $29(35,4)$ \\
\hline$*: p=0,001$ & &
\end{tabular}

analisadas, que três das respostas obtidas se inseriam em 'métodos de avaliação curricular', uma delas em 'exigências curriculares do MIM', e a outra correspondia a 'questões financeiras'.

\section{DISCUSSÃO}

Não sendo intenção deste estudo criar um instrumento psicométrico para medir o perfecionismo, nem comproválo, procurou-se então perceber, na ótica dos estudantes, o que está por detrás deste perfeccionismo e o que o influencia.

Para aplicação e divulgação do questionário, recorreuse a redes sociais através das comissões de curso do MIM-FMUC, ao invés da utilização de tecnologia de aleatorização para seleção dos participantes, ou aplicação do
Tabela 6 - Pressão Ambiental em função da Inserção numa atividade extracurricular

\begin{tabular}{|c|c|c|}
\hline & \multicolumn{2}{|c|}{$\begin{array}{l}\text { Inserção em atividade } \\
\text { extracurricular * }\end{array}$} \\
\hline & $\begin{array}{l}\text { Sim } \\
\text { n (\%) }\end{array}$ & $\begin{array}{l}\text { Não } \\
\text { n (\%) }\end{array}$ \\
\hline \multicolumn{3}{|l|}{ Pressão ambiental * } \\
\hline 1 - Nada importante & $12(5,7)$ & $8(5,0)$ \\
\hline 2 - Pouco importante & $68(32,5)$ & $29(18,2)$ \\
\hline 3 - Importante & $75(35,9)$ & $68(42,8)$ \\
\hline 4 - Muito importante & $54(25,8)$ & $54(34,0)$ \\
\hline
\end{tabular}

questionário em ambiente de sala de aula. Assim, esta amostragem de conveniência trouxe como desvantagens a perda de aleatoriedade, a maior dependência do voluntarismo e a impossibilidade de dominar o tamanho da amostra bem como de controlar quem responde. Todavia, a ágil aplicação, o mais fácil registo de dados e a resposta sem influência da pressão do ambiente de aula, com consequente eliminação dos vieses de disponibilidade e oportunidade, constituem vantagens desta metodologia, que permitiu ainda obter um tamanho de amostra adequado, de $n=368$, superior ao número necessário inicialmente estimado de $\mathrm{n}=277$ alunos. $\mathrm{O}$ facto de $77,7 \%$ da amostra pertencer ao sexo feminino reforça a mesma como representativa da 
população em estudo, estando em concordância com os dados de frequência do MIM-FMUC e da população de estudantes de medicina portugueses, constituída maioritariamente por mulheres. ${ }^{2,14}$

Os motivos elencados pelos alunos estão alinhados com a literatura ${ }^{7,8,13,14} \mathrm{e}$, neste estudo, mais de metade dos participantes considerou todos os motivos identificados como importantes para o seu perfeccionismo e intolerância à frustração. No entanto, o recurso a escala de tipo Likert pode ter originado o viés de concordância e, por outro lado, as classificações 'importante'/ 'muito importante' maioritariamente atribuídas poderão advir do perfil tipicamente perfeccionista desta população, um factor de confundimento que importa ter em consideração. ${ }^{14,16}$

Verificou-se que $33,4 \%$ da amostra não se encontra satisfeita com a vida curricular, sendo esse aspeto independente do ano de frequência do MIM, o que implica questionar se estes resultados estarão de facto ligados ao currículo, ${ }^{5-7,9}$ ou se se devem a fatores da própria personalidade exigente e perfeccionista desta população. ${ }^{7,9,14,16}$

Afirmando $56,8 \%$ da amostra estar inserida em algum tipo de atividade extracurricular, tal como em outros estudos nacionais, ${ }^{2,14}$ este valor está desalinhado das percentagens mais elevadas verificadas em trabalhos internacionais, ${ }^{3,9,17}$ o que leva a questionar se a carga curricular das escolas médicas portuguesas condiciona a falta de tempo para essas atividades, ,2,7,8,14 ou se tal se trata apenas de uma questão cultural ou social. A flutuação da inserção em atividades extracurriculares, verificada entre os diferentes anos, deverá ser estudada em trabalhos posteriores, supondo-se que a baixa proporção de alunos inseridos em alguma atividade no primeiro ano se deva ao período de adaptação inicial ${ }^{6}$ e à pressão sentida para manutenção do ritmo de aprendizagem adquirido no ensino secundário. ${ }^{2,5,16}$ Surgirá depois melhor adaptação ${ }^{4}$ e maior disponibilidade, traduzida pela maior percentagem de alunos com ocupação extracurricular nos anos subsequentes, que decresce após o quarto ano, provavelmente por aproximação da Prova Nacional de Seriação, com maior foco na vida curricular por perceção da necessidade de ser melhor que os outros para alcançar o futuro pretendido. ${ }^{13}$

Embora múltiplos estudos $4,6,7,9,12,13$ tenham demonstrado que as principais fontes de stress na formação médica são inerentes ao ambiente curricular, os presentes resultados apontam os traços de personalidade e o receio das exigências que a própria profissão médica acarreta, como os principais motivos para o perfeccionismo e intolerância à frustração nos estudantes do MIM-FMUC. Sabe-se que nos estudantes de medicina é frequente o perfeccionismo mal-adaptativo (neurótico), ${ }^{16}$ estando elevados níveis de neuroticismo ${ }^{8,13}$ e evicção do perigo (harm avoidance ${ }^{20} \mathrm{e}$ baixos níveis de autodeterminação (self-directedness) ${ }^{20}$ associados a maior vulnerabilidade ao stress. Por outro lado, o facto da profissão médica obrigar a lidar com questões de extrema sensibilidade ${ }^{13}$ e não permitir lugar para o erro, apesar da permanente incerteza, ${ }^{13,18}$ torna este aspeto numa igual fonte de stress, tanto para os profissionais como para os alunos, que parecem sofrer por antecipação.

A 'pressão ambiental' é considerada o motivo menos importante pela amostra, embora na literatura a formação médica seja apontada, por si só, como um ambiente de extrema pressão, 8,11,13 "que encoraja a competição no lugar da cooperação entre os colegas". ${ }^{11}$

Os restantes motivos mostraram também representar um importante papel no perfeccionismo e intolerância à frustração nestes estudantes, com $80,4 \%$ a $84,8 \%$ da amostra a tomá-los como 'importante'/'muito importante', observando-se que os alunos não satisfeitos com a vida curricular Ihes atribuíam maior importância. Assim, esta variável deverá ser abordada em novos estudos, que reúnam informação sobre as causas da insatisfação curricular, assim permitindo propor reestruturações curriculares que otimizem o equilíbrio entre o bem-estar dos alunos e a manutenção de um ensino médico de qualidade. ${ }^{12}$

Os alunos inseridos numa atividade extracurricular parecem dar menor importância à 'pressão ambiental', de onde se conclui que, provavelmente, as atividades extracurriculares desempenham um papel protetor, pelo desenvolvimento de capacidades que ajudam a lidar melhor com pressões ou dificuldades. ${ }^{9}$

Este estudo, ao compreender os principais motivos por detrás da vulnerabilidade ao stress psicológico nos estudantes do MIM-FMUC, promove o pensar de táticas dirigidas à prevenção de tal vulnerabilidade, nomeadamente o desenvolvimento de capacidade organizativa e de aquisição de métodos de estudo logo no ensino secundário ${ }^{19}$; a formação dos alunos acerca do stress psicológico ${ }^{7,13}$ e seu alívio ${ }^{1,5,8}$; a disponibilização e facilitação de suporte psicossocial ${ }^{1,7}$ por parte de alunos mais velhos, ${ }^{5}$ professores, psicólogos ou colegas, incluindo a promoção de atividades de grupo e de programas curriculares/extracurriculares que promovam a cooperação entre alunos ${ }^{13}$; a formação dos professores e assistentes para estratégias educativas, preventivas do stress negativo e promotoras do bem-estar e adequada aprendizagem dos alunos ${ }^{13}$; a partilha de experiências por parte de médicos séniores,,$^{18}$ focando a incerteza presente em todos os atos da profissão numa perspetiva humanista e contemporânea da medicina, ${ }^{7}$ e mesmo técnicas cognitivas comportamentais positivas. ${ }^{21}$

O perfeccionismo socialmente prescrito, que poderá comprometer a auto-eficácia académica e estar na base do burnout em estudantes e em futuros médicos, ${ }^{22}$ não foi por nós abordado, considerando-se relevante a sua exploração em futuros trabalhos. Por outro lado, a transversalidade deste estudo, que não permite atribuir causalidade, justifica a realização de estudos posteriores longitudinais, alargados a outras amostras e/ou populações dentro do universo dos estudantes de medicina portugueses.

\section{CONCLUSÃO}

Concluíram-se como principais motivos para o perfeccionismo e intolerância à frustração nos estudantes do MIM-FMUC, os 'fatores intrínsecos' dependentes da personalidade, e as 'exigências da profissão médica'. 
Seguem-se 'incerteza quanto ao futuro pretendido', 'insegurança quanto à formação profissional', 'métodos de avaliação curricular' e 'exigências curriculares do MIM'. A 'pressão ambiental' é o fator de menor importância, ainda que, para mais de metade da amostra estudada, todos os motivos identificados sejam relevantes.

O peso dos diferentes fatores não varia consoante o ano de frequência do MIM-FMUC. Já a 'insegurança quanto à formação profissional', os 'métodos de avaliação curricular' e as 'exigências curriculares do MIM' são considerados menos importantes pelos alunos satisfeitos com a vida curricular, sendo que a 'pressão ambiental' apresenta menor relevância não só para estes alunos, como também para os inseridos em atividades extracurriculares.

Pretende-se que, com base nos resultados apresentados e discutidos, se levem a cabo intervenções, por parte da Faculdade de Medicina da Universidade de Coimbra, que previnam os motivos identificados, e que as mesmas sirvam como exemplo às restantes escolas médicas do país.

\section{REFERÊNCIAS}

1. IsHak W, Nikravesh R, Lederer S, Perry R, Ogunyemi D, Bernstein C. Burnout in medical students: a systematic review. Clin Teach. 2013;10:242-5.

2. Loureiro E, Mcintyre T, Mota-Cardoso R, Ferreira MA. A relação entre o stress e os estilos de vida nos estudantes de medicina da Faculdade de Medicina do Porto. Acta Med Port. 2008;21:209-14.

3. Fares J, Saadeddin Z, Al Tabosh H, Aridi H, El Mouhayyar C, Koleilat MK, et al. Extracurricular activities associated with stress and burnout in preclinical medical students. J Epidemiol Glob Health. 2016;6:177-85.

4. Yee LY, Yusoff MS. Prevalence and sources of stress among medical students in Universiti Sains Malaysia and Universiteit Maastricht. Educ Med J. 2013;5:34-41.

5. Fares J, Al Tabosh H, Saadeddin Z, El Mouhayyar C, Aridi H. Stress, burnout and coping strategies in preclinical medical students. N Am J Med Sci. 2016;8:75-81.

6. Heinen I, Bullinger M, Kocalevent RD. Perceived stress in first year medical students - associations with personal resources and emotional distress. BMC Med Educ. 2017;17:1-14.

7. Khapre M, Mudey A, Nayak S, Goyal RC. Assessment of stressors in medical students and its relationship with the self-rated depression. Int J Curr Res Rev. 2014;6:5- 9.

8. Bugaj TJ, Cranz A, Junne F, Erschens R, Herzog W, Nikendei C. Psychosocial burden in medical students and specific prevention strategies. Ment Health Prev. 2016;4:24-30.

9. Yusoff MS, Abdul Rahim AF, Baba AA, Ismail SB, Mat Pa MN, Esa AR. Prevalence and associated factors of stress, anxiety and depression among prospective medical students. Asian J Psychiatr. 2013;6:128-33.

10. Singh S, Prakash J, Das RC, Srivastava K. A cross-sectional assessment of stress, coping, and burnout in the final-year medical undergraduate students. Ind Psychiatry J. 2016;25:179-83.

11. El-Masry R, Ghreiz SM, Helal RM; Audeh AM, Shams T. Perceived

\section{PROTECÇÃO DE PESSOAS E ANIMAIS}

Os autores declaram que os procedimentos seguidos estavam de acordo com os regulamentos estabelecidos pelos responsáveis da Comissão de Investigação Clínica e Ética e de acordo com a Declaração de Helsínquia da Associação Médica Mundial.

\section{CONFIDENCIALIDADE DOS DADOS}

Os autores declaram ter seguido os protocolos do seu centro de trabalho acerca da publicação de dados.

\section{CONFLITOS DE INTERESSE}

Os autores declaram não terem qualquer conflito de interesse relativamente ao presente artigo.

\section{FONTES DE FINANCIAMENTO}

Os autores declaram não ter recebido subsídios ou bolsas para a elaboração do artigo.

stress and Burnout among medical students during the clinical period of their education. Ibnosina J Med BS. 2013;5:179-87.

12. Yusoff MS, Abdul Rahim AF, Baba AA, Ismail SB, Mat Pa MN, Esa AR. The impact of medical education on psychological health of students: a cohort study. Psychol Health Med. 2013;18:420-30.

13. Dyrbye L, Shanafelt T. A narrative review on burnout experienced by medical students and residentes. Med Educ. 2016;50:132-49.

14. Pereira AP. Qualidade de vida e vulnerabilidade ao stress nos estudantes de medicina dos $5^{\circ}$ e $6^{\circ}$ anos. Tese (Mestrado Integrado em Medicina). Coimbra: Universidade de Coimbra; 2017.

15. Vaz Serra A. Construção de uma escala para avaliar a vulnerabilidade ao stress: a 23 QVS. Psiquiatr Clin. 2000;21:297-308.

16. Enns W, Cox B, Sareen J, Freeman P. Adaptive and maladaptive perfectionism in medical students: a longitudinal investigation. Med Educ. 2001;35:1034-42.

17. Almalki SA, Almojali Al, Alothman AS, Masuadi EM, Alaqeel MK. Burnout and its association with extracurricular activities among medical students in Saudi Arabia. Int J Med Educ. 2017;8:144-50.

18. Benbassat J, Baumal R, Chan S, Nirel N. Sources of distress during medical training and clinical practice: Suggestions for reducing their impact. Med Teach. 2011;33:486-90.

19. Barbosa J, Ferreira MA, Severo M, Silva Á. Transition from secondary school to medical school: the role of self-study and self-regulated learning skills in freshman burnout. Acta Med Port. 2016;29:803-8.

20. Lee $\mathrm{S}$, Choi $\mathrm{Y}$, Chae $\mathrm{H}$. The effects of personality traits on academic burnout in Korean medical students. Integr Med Res. 2017;6:207-13.

21. Chand S, Chibnall J, Slavin S. Acad Psychiatr. 2018;42:58-61.

22. $\mathrm{Yu} \mathrm{JH}$, Chae SJ, Chang $\mathrm{KH}$. The relationship among self-efficacy, perfectionism and academic burnout in medical school students. Korean J Med Educ. 2016;28:49-55. 